\title{
Glosse
}

\section{Monika Frommel \\ Die Juniorprofessur - ist das die frauenfreundliche Alternative zur alten Ordinarienherrlichkeit?}

Künftig wird es keine Habilitation mehr geben. Da sie als Prüfungsleistung gilt, kann (und will) das (künftige) Hochschulrecht es verbieten, diese Qualifikation bei Einstellungen positiv zu werten. Es geht also nicht um mehr Pluralität auf dem Wege zur Professur, sondern um mehr Regulierung, und das in einem Feld, das ohnehin fast totreguliert ist. Selbst wenn sie es gerne anders haben wollen, müssen sich künftig alle NachwuchswissenschaftlerInnen um die begehrten Juniorprofessuren bewerben und das volle Lehrdeputat erfahrener KollegInnen - mit allen damit verbundenen Evaluationen - absolvieren. Ein zweites Buch wird bis zur endgültigen Einstellung freilich erwartet. Aber ob es für gut befunden wird, entscheidet nicht mehr die abgebende Hochschule, sprich der Mentor, der die Arbeit betreut hat (Habilmütter sind selten), sondern es wird in einem staatlich kontrollierten, mehrstufigen Bewertungsverfahren von der aufnehmenden Hochschule entschieden, ob der oder die Juniora würdig ist, kooptiert zu werden. Eines ist sicher: Der Anpassungsdruck wird höher, nicht geringer. Graue Mäuse gegen bunte Vögel. Das alte System mag manchmal ärgerlich gewesen sein. Aber es privilegierte zumindest in den Nischen Innovationen. In Zeiten der Not werden aber als erstes die Nischen abgeschafft. Dafür werden Modethemen etabliert: in den 8oer Jahren ein wenig Frauenforschung, heute MultiMedia, morgen Gentechnologie. Klar - die ewig Gestrigen meiner Zunft regen sich auf, weil eine heilige Kuh geschlachtet wird. Klar ist auch, daß die jungen Kollegen, die sich gerade habilitiert haben oder dabei sind, es zu tun, ihre Rechte schwer verletzt sehen. Schließlich werden sie auf dem Altar der Modernisierung ohne Blick zurück und ohne Öffnungsklausel geopfert. Nur noch ärgerlich finde ich aber, daß ausgerechnet Frauenpolitikerinnen sich einbilden, die Abwertung der ganzen Laufbahn wirke frauenfördernd (Förderung im Abwärtstrend!).

Ich möchte einmal quer zu dem bekannten Meinungsspektrum fragen, was der Effekt sein wird?

Die neue Hochschulstruktur führt bei den Rechtswissenschaften und den Geisteswissenschaften zu einer Nivellierung, die den Namen Reform nicht verdient. Wenn man sparen muß, dann bitte überlegt und nicht über eine Abwertung der ganzen Laufbahn. Die Orientierung an der Drittmittelforschung passt zu naturwissenschaftlicher Forschung und führt auch in den medizinischen Fakultäten zu sozialverträglicheren Laufbahnen. Aber sie fördert weder Frauen noch ist sie die Lösung des Problems.

Fragen wir, wie alles anfing: Eine Generation (relativ) gut dotierter Kollegen freut sich auf die Pension, ihr Ruhegehalt belastet die Länder, sie müssen sparen, wenn sie nicht kurzfristig Hochschulen schließen wollen, weil die Lehre nicht mehr gesichert ist. Forschung findet daher nur statt, wenn Drittmittel eingeworben werden. Ein für Kliniken, Natur- und Sozialwissenschaften sinnvoller Weg, aber ansonsten sehe ich Sackgassen. Für die Geistes- und Rechtswissenschaften wird es finster. 
Verkauft wird das Ganze nach der Logik der Weißwäscher: Die Abschaffung der oldboys-network fördere Frauen. Endlich versetze man der alten Ordinarienherrlichkeit den entscheidenden Schlag. Das soll es gewesen sein? Ich reibe mir die Augen und schaue die triste Realität an, unter der Hochschulen in ärmeren, meist sozialdemokratisch regierten Ländern zu leiden haben. Sie müssen mit Unterfinanzierungen rechnen, die in der freien Wirtschaft allenfalls kurz vor der Pleite ertragen werden. Außer in Bayern und Baden-Württemberg wird gerade mal das Gehalt gezahlt, ansonsten wird von der Substanz gelebt und der Mangel mit immer mehr Bürokratie verwaltet. Deren Kosten steigen: Mängelverwaltung und Planwirtschaft scheinen sich nämlich bestens zu vertragen. Reformen von oben verlangen Evaluation des $\mathrm{Be}-$ stehenden und Steuerung durch Vorschriften. Gigantische Verwaltungen nehmen uns Lehrenden und Forschenden nicht Verwaltungsarbeit ab, sondern vermehren sie. Nicht das Unternehmen und schon gar nicht die Produkte der trotz aller Widrigkeiten betriebenen "Forschung und Lehre« sind zu teuer, sondern der aufgeblähte Apparat der Zentralverwaltungen. Absprachen und Vorschriften auf Hochschulebene, in den Landes- und dem Bundesministerium, abgestimmt mit Verbänden, überregionalen Gremien, erörtert auf Tagungen. Berge von Papieren türmen sich wie in einer chaotischen Planwirtschaft. Ich sehne mich nach einem kontrollierten Markt. Denn was zur Zeit als Modernisierung verkauft wird, ist jedenfalls keine Liberalisierung und Dezentralisierung. Nur der Mangel wird dezentralisiert, da die Kosten der Planwirtschaft zu Einsparungen bei den eigentlichen Aufgaben zwingen. Kurzfristige und insgesamt völlig chaotische Sparbeschlüsse, die je nach Haushaltslage mal so, mal anders ausfallen, gefährden bereits eine angemessene Lehre und Forschung. Wir leben seit Jahren mit Wiedereinstellungssperren. Sie verhindern eine gezielte Nachwuchspolitik und wirken viel willkürlicher als frühere Ordinarienwillkür je wirken konnte. Sicher, damals konnte über Fristverträge Druck auf Mitarbeiter ausgeübt werden. Aber heute kann man den künftigen Wissenschaftlern selbst bei bestem Willen die ökonomische Sicherheit gar nicht mehr bieten, die wir, damit meine ich die jetzigen HochullehrerInnen, noch hatten. Innovative Leistungen waren im Rahmen der alten Strukturen einer Ordinarienuniversität durchaus möglich. Wer etwas mutig und durchsetzungsfähig war, konnte in den letzten drei Jahrzehnten einen Mentor finden, der seine Freiheit zum Nutzen seiner MitarbeiterInnen zu nutzen verstand. Jedenfalls habe ich viele, durchaus auch Konservative kennen gelernt, die damit keine Probleme hatten. Es gibt keinen Grund anzunehmen, daß der Marsch durch die Institutionen im universitären Milieu weniger erfolgreich gewesen sein soll als in der Politik. In beiden Szenen gibt es ewig Gestrige, aber im Amt sind zur Zeit diejenigen, die als Studenten noch für Reformen gekämpft haben. Eine ganze Generation mehr oder weniger liberaler Hochschullehrer wird gerade emeritiert. Sie haben die zweite oder dritte Demokratisierung getragen. Daß es in ihrer Generation besonders wenige Frauen gewagt haben, hat viele Gründe: Aber sie scheinen mir in ihrer Bedeutung nachzulassen. Viele Frauen haben in der Vergangenheit nicht habilitiert, weil der Arbeitsplatz Uni für sie weniger interessant war als andere. Heute könnte eine Universitätslaufbahn wieder interessant sein, da Frauen mittlerweile bessere Netzwerke zu ihrer Unterstützung vorfinden - zumindest in den Geistes- und Sozialwissenschaften. Manchmal brauchen Veränderungen einfach etwas mehr Zeit. Kurzatmige Reformpolitik hingegen lähmt oder wirkt bestenfalls paradox. Ausgerechnet jetzt wird eine Personalstruktur geschaffen, die es aus meiner Sicht Frauen wesentlich schwerer macht, als sie es zu meiner Zeit hatten. Die formale Hürde der Habilitation kann eine motivierte Wissenschaftlerin nämlich nehmen - auch und gerade mit Kindern-, ein mehrstufiges Evaluationsverfahren hingegen, das Lehrtätigkeit, Drittmitteleinwerbung und Schriftenverzeichnis unter die Lupe nimmt, setzt so viel Bereitschaft 
zur Selbstausbeutung voraus, daß ich es mir nur mit einem Partner im Rücken, der die klassische Frauenrolle übernimmt, zutrauen würde. Gegen die verlangten Mühen spricht jede Vernunft. Die reformierte Laufbahn ist nicht mehr attraktiv - auf diese öde Logik läuft es hinaus; jedenfalls dort, wo Frauen zur Zeit arbeiten: in den Geistesund Sozialwissenschaften.

Wieso öffnet man die Qualifikationen nicht in Richtung habilitationsgleiche Leistung. Wozu noch ein erstickendes Fragebogen- und Beurteilungswesen? Der dirigistische Stil paßt zu den Berührungsängsten insbesondere sozialdemokratischer Wissenschaftsministerinnen vor den angeblich so elitären Universitäten. Sie paßt auch zu ihrer Neigung, alle möglichst zu Fachhochschulen herunter zu stufen, was mich fatal an nachgereichten Widerstand erinnert. Das verblaßte Feindbild des Altnazis im hohen Amt, der schöne Spruch vom Muff unter den Talaren, verdeckt, daß man sich auch zu Tode siegen kann.

Die Ordinarienherrlichkeit ist tot, aber die UNIVERSITAS leider auch. Drittmittelforschung kann doch nicht die Alternative sein!

Norbert Haase/Bert Pampel (Hrsg.)

\section{Die Waldheimer ,Prozesse ${ }^{66}$ - fünfzig Jahre danach}

\section{Dokumentation der Tagung der Stiftung Sächsische Gedenkstätten am 28. und 29. September 2000 in Waldheim}

Unter direkter Anleitung der SED-Führung verurteilten linientreue Richter zwischen Ende April und Mitte Juli 1950 in der sächsischen Stadt Waldheim mehr als 3300 Menschen zu langjährigen Haftstrafen. Die Verfahren widersprachen elementaren Rechtsgrundsätzen. Bei den Verurteilten handelte es sich mehrheitlich um Menschen, die in der Nachkriegszeit von der sowjetischen Geheimpolizei allein wegen ihrer Mitgliedschaft oder Funktion in der NSDAP oder in einer der ihr angeschlossenen Organisationen verhaftet worden waren.

50 Jahre nach den Waldheimer »Prozessen« beleuchten Historiker, Politologen und Juristen den Forschungsstand der letzten Jahre zusammenfassend. Dabei kommen verschiedene Aspekte dieses frühen Kapitels der DDR-Justizgeschichte, z. B. die Steuerung durch die SED-Führung oder die strafrechtliche Aufarbeitung nach 1989, zur Sprache. Sie ordnen die Verfahren in den Kontext der Auseinandersetzung mit den Verbrechen des Nationalsozialismus in der DDR ein und erörtern Perspektiven für den künftigen Umgang mit dem historischen Ort.

Die Tagungsdokumentation richtet sich an interessierte Historiker, Politikwissenschaftler und Juristen.

2001, XIV, 122 S., brosch., 48,- DM, 43,-sFr, ISBN 3-7890-7420-9

(Juristische Zeitgeschichte. Abteilung 2; Forum Juristische Zeitgeschichte, Beiträge und Berichte aus dem Arbeitskreis Juristische Zeitgeschichte, Bd. 11) 